\title{
A Secure Obfuscator for Encrypted Blind Signature Functionality $^{\star}$
}

\author{
Xiao Feng ${ }^{1,2}$ Zheng Yuan ${ }^{1,2 \star \star}$ \\ 1 Beijing Electronic Science \& Technology Institute, Beijing 100070, P. R. China \\ zyuan@tsinghua.edu.cn and yuanzheng@besti.edu.cn \\ 2 School of Telecommunications Engineering, Xidian University, Shaanxi 710071,P. R. China
}

\begin{abstract}
This paper introduces a new obfuscation called obfuscation of encrypted blind signature. Informally, Alice is Signer and Bob is User. Bob needs Alice to sign a message, but he does not want Alice to know what the message is. Furthermore, Bob doesn't want anyone to know the interactive process. So we present a secure obfuscator for encrypted blind signature which makes the process of encrypted blind signature unintelligible for any third party, while still keeps the original encrypted blind signature functionality. We use schnorr's blind signature scheme and linear encryption scheme as blocks to construct a new obfuscator. Moreover, we propose two new security definition: blindness w.r.t encrypted blind signature (EBS) obfuscator and one-more unforgeability(OMU) w.r.t EBS obfuscator, and prove them under Decision Liner Diffie-Hellman(DL) assumption and the hardness of discrete logarithm, respectively. We also demonstrate that our obfuscator satisfies the Average-Case Virtual Black-Box Property(ACVBP) property w.r.t dependent oracle, it is indistinguishable secure. Our paper expands a new direction for the application of obfuscation.
\end{abstract}

Keywords: Obfuscation, Blind signature, Indistinguishable security.

\section{Introduction}

Obfuscation in cryptography has been formally proposed by Barak, Goldreich et al.[1] at the first time. Although it is a theoretical hotspot, there hasn't been much progress in recent years. The implementation of obfuscation mainly depends on how to construct a secure obfuscator. Informally, obfuscator is an algorithm program which can transform a program into a new unintelligible program while its functionality holds. Barak et al. suggested that an obfuscator should satisfy the following three properties:

1. Functionality: the obfuscated program has the same functionality as the original program.

2. Polynomial Slowdown: the description length and running time of the obfuscated program are at most polynomially larger than the original program's.

\footnotetext{
* This work is supported by the National Natural Science Foundation of China (No.61070250), and Beijing Natural Science Foundation (N0.4132066), and the 12th Five-year Cryptography Development Foundation of China (No.MMJJ201101026), and Scientific Research and Postgraduate Training Cooperation Project-Scientific Research Base-New Theory of Block Cipher and Obfuscation and their Application Research.

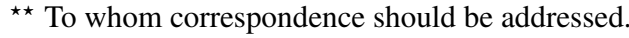


3. Virtual Black-Box Property(VBP): Anything that can be efficiently computed from obfuscated program can be efficiently computed given oracle access to the original program.

Obfuscation has profound effects on both theory and application, such as software protection, homomorphic encryption, removing random oracles and transforming private-key encryption into public-key encryption. Despite all that, Barak et al. proved the impossibility of obfuscation even under a very weak definition. Later, more impossible obfuscation results of natural functionalities were shown in [2][3][4] [5]. Even so, cryptographic communities has been dedicating to conduct a series of explorations, and they found that there still exist simple classes of functions such as point functions [6][7][8][2][3][9] with the possibility of obfuscation.

Before 2007, several positive results of obfuscation were mainly about simple functions. The obfuscation of complicated cryptographic functionality was firstly proposed by Hohenberger et al.[10] in TCC'07. They obfuscated re-encryption and proved the security of obfuscator in the standard model. In brief, the re-encryption functionality is the one that takes a ciphertext for a message encrypted under Alice's public key and transforms it into a ciphertext for the same message under Bob's public key. Hohenberger et al. presented an improved security property called ACVBP. Following the security definition of ACVBP[10], Hada [11] showed a secure obfuscation for encrypted signature, which generated a signature on a given message under Alice's secret signing key and then encrypted the signature under Bob's public encryption key. Later, on the basis of Honhenberger's results, Nishanth Chandran et al. [12] refined the delegation of access of re-encryption functionality, and demonstrated the security of collusion-resistant obfuscation. These are the only known three obfuscations of complicated cryptographic functionality.

Blind signature has a wide range of applications in e-cash and electronic election. A blind signature is a protocol introduced by Chaum [13] for protecting the anonymity of signer, which was based on the RSA digital signature scheme. Unlike general digital signature scheme, blind signature requires that the signer signs the message without knowing the message or the resulting signatures while the user can verify it publicly. It's an interactive protocol between the signer and user. A blind signature must satisfy the following properties:

1. Unforgeability: Adversary can not produce a legal blind signature on message after interacting with signer.

2. Blindness: The signatures of two given messages are computationally indistinguishable even under a set of known message-signature pairs.

Afterwards, on the basis of Schnorr's signature scheme, Okamoto[14] put forward a blind signature scheme named Schnorr's blind signature whose security was based on discrete logarithm problem. Schnorr[15] then proved its security.

In this paper, we firstly use Schnorr's blind signature scheme and linear encryption scheme [16] as blocks to construct a secure obfuscator for blind signature, which is complete and verifiable. In order to prove the security of the obfuscator, we propose two new security definitions, Blindness w.r.t encrypted blind signature(EBS) obfuscator and one-more unforgeability (EBS) obfuscator, to prove Theorem 5. The main method 
is constructing different adversaries to break the hardness assumption under security definition of ACVBP w.r.t dependent oracle, the scheme is insecure if any adversary succeeds. The specific progress refers to section 5. We also prove that the OMU w.r.t EBS functionality implies OMU w.r.t EBS obfuscator under the assumption that EBS obfuscator satisfies ACVBP w.r.t dependent oracle set. Obviously, we have OMU w.r.t EBS obfuscator. At last, we present the security proof of EBS obfuscator. i.e., the EBS obfuscator satisfies ACVBP w.r.t dependent oracle. Thus, we illustrate that under the ACVBP w.r.t dependent oracle, generating a blind signature on a message and then encrypting the signature are functionally equivalent to encrypt the sign key and then generate a blind signature on the message.

The paper is organized as follows: Section 2 gives preliminaries which contain three parts; Section 3 proposes new security definitions with respect to the basis of theorem's proof; Then section 4 constructs the secure obfuscator for special EBS functionality and section 5 gives the proof.

\section{Preliminaries}

In this section, we present the basic security definition and the hardness assumption that our proofs rely on.

\subsection{Bilinear Maps}

Set BMsetup be an initialization algorithm: on input security parameter $1^{k}$, outputs the bilinear map parameters as $\left(q, g, \mathbb{G}, \mathbb{G}_{T}, \mathbf{e}\right)$, where $\mathbb{G}, \mathbb{G}_{T}$ are groups of prime order $q \in \Theta\left(2^{k}\right), g$ is a generator of $\mathbb{G}$ and $\mathbf{e}$ is an efficient bilinear mapping from $\mathbb{G} \times \mathbb{G}$ to $\mathbb{G}_{T}$. The mapping e satisfies the following two property:

- Bilinear: For all $g \in \mathbb{G}$ and $a, b \in \mathbb{Z}_{q}, \mathbf{e}\left(g^{a}, g^{b}\right)=\mathbf{e}(g, g)^{a b}$.

- Non-degenrate: If $g$ generates $\mathbb{G}$, then $\mathbf{e}\left(g^{a}, g^{b}\right) \neq 1$.

\subsection{Complexity Assumptions}

Definition 1. (DL Assumption) For every PPT machine D, every polynomial $p(\cdot)$, all sufficiently large $n \in \mathbb{N}$, and every $z \in\{0,1\}^{\operatorname{ploy}(n)}$,

$$
\begin{aligned}
& \mid \operatorname{Pr}\left[\begin{array}{l}
p=\left(q, \mathbb{G}, \mathbb{G}_{T}, \boldsymbol{e}, g\right) \leftarrow \operatorname{Setup}\left(1^{n}\right) ; \\
a \leftarrow \mathbb{Z}_{q} ; b \leftarrow \mathbb{Z}_{q} ; r \leftarrow \mathbb{Z}_{q} ; s \leftarrow \mathbb{Z}_{q} ; \\
\text { decision } \leftarrow D\left(p,\left(g^{a}, g^{b}\right),\left(g^{r+s},\left(g^{a}\right)^{r},\left(g^{b}\right)^{s}\right), z\right) .
\end{array}\right]- \\
& \operatorname{Pr}\left[\begin{array}{l}
p=\left(q, \mathbb{G}, \mathbb{G}_{T}, \boldsymbol{e}, g\right) \leftarrow \operatorname{Setup}\left(1^{n}\right) ; \\
a \leftarrow \mathbb{Z}_{q} ; b \leftarrow \mathbb{Z}_{q} ; r \leftarrow \mathbb{Z}_{q} ; s \leftarrow \mathbb{Z}_{q} ; t \leftarrow \mathbb{Z}_{q} ; \\
\text { decision } \leftarrow D\left(p,\left(g^{a}, g^{b}\right),\left(g^{t},\left(g^{a}\right)^{r},\left(g^{b}\right)^{s}\right), z\right) .
\end{array}\right] \mid \leqslant \frac{1}{p(n)}
\end{aligned}
$$




\subsection{The Definition of General Security}

In this subsection we review the security definition of public-key encryption(PKE) scheme and digital blind signature(DBS) scheme. Setup is an algorithm that generates a parameter, on security parameter $1^{n}$, which is used commonly by multiple users in a pair of $P K E$ and $D B S$ schemes.

A probabilistic public key cryptosystem $P K E$ is a probabilistic polynomial time Turing machine $\Pi$ that

(1)EKG: on inputs $p$ generates a pair of pubic-secret key $(p k, s k)$ and outputs the description of two algorithms, $E$ and $D$ such that

(2) $E$ is a probabilistic encryption algorithm: for some constants $p$, public key $p k$ and a plaintext $m$, returns the ciphertext $c$, let $M S(p, p k)$ be the message space defined by $(p, p k)$.

(3) $D$ is a deterministic decryption algorithm: for some constants $p$, secret key sk and ciphertext $c$, returns the plaintext $m$.

Definition 2. (Indistinguishability of Encryptions against CPAs) A PKE scheme (EKG, $E, D)$ satisfies the indistinguishability if the following condition holds: For every PPT machine pair $\left(A_{1}, A_{2}\right)$ (adversary), every polynomial $p(\cdot)$, all sufficiently large $n \in \mathbb{N}$, and every $z \in\{0,1\}^{\text {poly }(n)}$,

$$
2 \cdot \operatorname{Pr}\left[\begin{array}{l}
p \leftarrow \operatorname{Setup}\left(1^{n}\right) ;(p k, s k) \leftarrow E K G(p) ; \\
\left(m_{1}, m_{2}, h\right) \leftarrow A_{1}(p, p k, z) ; b \leftarrow\{0,1\} ; c \leftarrow E\left(p, p k, m_{b}\right) ; \\
d \leftarrow A_{2}\left(p, p k,\left(m_{1}, m_{2}, h\right), c, z\right) ; \\
b=d .
\end{array}\right]-1 \leqslant \frac{1}{p(n)}
$$

where we assume that $A_{1}$ produces a valid message pair $m_{1}$ and $m_{2} \in M S(p, p k)$ and a hints $h$.

A digital blind signature $D B S$ also contains three algorithms:

(1) $S K G$ : generates a pair of pubic-secret key $(p k, s k)$ on input $p$.

(2) $(S, U)$ is a probabilistic interactive signing algorithm: for some constants $p$, secret key $s k$ and $l$-bit plaintext $m=m_{1} m_{2} \cdots m_{l} \in M S(p, p k)$, the execution of algorithm $S(s k)$ (by signer), and algorithm $U(p k, m)$ (by user) for message $m$ generates the signature $\sigma$, where $M S(p, p k)$ is the message space defined by $(p, p k)$.

(3) $V$ is a deterministic verification algorithm: for some constants $p$, public key $p k$, message $m$ and signature $\sigma$, if $\sigma$ is the valid signature of $m$, it accepts; Otherwise returns $\perp$.

The security of a blind signature scheme includes one-more unforgeability and blindness.

Definition 3. (Blindness) A blind signature scheme DBS $=(S K G,(S, U), V)$ is called blind if for any efficient algorithm $A_{3}$, all sufficiently large $n \in \mathbb{N}$, and every $z \in$ $\{0,1\}^{\text {poly }(n)}$, there exists

$2 \cdot \operatorname{Pr}\left[\begin{array}{l}p \leftarrow \operatorname{Setup}\left(1^{n}\right) ;(p k, s k) \leftarrow S K G(p) ; \\ b \leftarrow\{0,1\} ;\left(\sigma_{0}, \sigma_{1}\right) \leftarrow A_{3}^{<\cdot, U\left(p k, m_{b}\right)>^{1},<\cdot, U\left(p k, m_{1-b}\right)>^{1}}(p, p k, z) ; \\ b^{*} \leftarrow A_{3}\left(\sigma_{0}, \sigma_{1}\right) ; \\ b=b^{*} .\end{array}\right]-1 \leqslant \frac{1}{p(n)}$ 
where $A_{3}$ is the malicious Signer and $U$ is the honest user. If $\sigma_{0}=\perp$ or $\sigma_{1}=\perp$, then the Signer is not informed about the other signature.

Note that we use $X^{<\cdot, Y\left(y_{0}\right)>^{1},<\cdot, Y\left(y_{1}\right)>^{1}}$ to define the process that $X$ invokes arbitrarily ordered executions with $Y\left(y_{0}\right)$ and $Y\left(y_{1}\right)$, but interacts with each algorithm only once.

Definition 4. (One-more Unforgeability) A DBS scheme ( $S G,(S, U), V)$ is unforgetable if for any efficient algorithm $A_{4}($ the malicious user), every polynomial $p(\cdot)$, all sufficiently large $n \in \mathbb{N}$, and every $z \in\{0,1\}^{\text {poly }(n)}$, there exist

$$
\operatorname{Pr}\left[\begin{array}{l}
p \leftarrow \operatorname{Setup}\left(1^{n}\right) ;(p k, s k) \leftarrow S K G(p) ; \\
\left(\left(m_{1}^{*}, \sigma_{1}^{*}\right), \ldots,\left(m_{k+1}^{*}, \sigma_{k+1}^{*}\right)\right) \leftarrow A_{4}^{\left.\ll S_{p, s k}\right\rangle^{k}}(p, p k, z) ; \\
\text { if } m_{i}^{*} \neq m_{j}^{*} \text { for } i \neq j ; \\
V\left(p, p k, m_{i}^{*}, \sigma_{i}^{*}\right)=\text { Accept for all } i \text {; then return } 1 .
\end{array}\right] \leqslant \frac{1}{p(n)}
$$

where $S_{p, s k}$ is the signing oracle (circuit).

Note that we use $X^{\ll Y \gg^{k}}$ to define the process that $X$ samples access to $Y$ for at most $k$ times.

\section{Construct the Secure Obfuscator for Special EBS Functionality}

This section presents a secure obfuscator for the blind signature and proves the security based on the generalized ACVBP definition.

\subsection{Schnorr's Blind Signature}

We use Schnorr's blind signature scheme[14] as a block to build the EBS functionality. The specific process is as follows:

$S K G(p)$

1. Parses $p=\left(q, \mathbb{G}, \mathbb{G}_{T}, \mathbf{e}, g\right)$.

2. Selects $g_{1} \in \mathbb{G}$ and $x \in \mathbb{Z}_{q}$ randomly.

3. Outputs the secret key $s k=g_{1}^{x}$ and public key $p k=\left(g_{1}, g^{g_{1}^{x}}\right)$, where $y=g^{g_{1}^{x}}$.

$\operatorname{Sign}(p, s k, m)$

1. Parses $p=\left(q, \mathbb{G}, \mathbb{G}_{T}, \mathbf{e}, g\right)$.

2. Signer selects $k \in \mathbb{Z}_{q}$ randomly and computes $t=g^{k} \bmod p$, then sends $t$ to User.

3. User selects $\alpha, \beta \in \mathbb{Z}_{q}$ randomly and computers $\omega=\operatorname{tg}^{\alpha} y^{\beta} \bmod p$, then computes $c=H(m \| \omega)$ and $c^{\prime}=c-\beta \bmod q$, sends $c^{\prime}$ to Signer.

4. Signer computes $u=k-c^{\prime} \cdot \operatorname{sk} \bmod q$, and sends $u$ to User.

5. User computes $v=u+\alpha \bmod q$.

6. User outputs signature $\sigma=(c, v)$.

$\operatorname{Verify}(p, p k, m, \sigma)$

1. Parses $p=\left(q, \mathbb{G}, \mathbb{G}_{T}, \mathbf{e}, g\right), p k=\left(g_{1}, g^{g_{1}^{x}}\right), m=m_{1}, m_{2}, \ldots, m_{n}$, and $\sigma=(c, v)$.

2. Computes $g^{v} y^{c}=\omega$.

3. Accepts if $H(m \| \omega)=c$; otherwise outputs $\perp$. 


\subsection{Linear Encryption Scheme}

Boneh's linear encryption scheme[16] is another block to build the EBS functionality. The detail is as follows:

$E K G(p)$ :

1. Parses $p=\left(q, \mathbb{G}, \mathbb{G}_{T}, \mathbf{e}, g\right)$.

2. Selects $a \in \mathbb{Z}_{q}$ and $b \in \mathbb{Z}_{q}$ randomly.

3. Outputs the secret key $s k_{e}=(a, b)$ and public key $p k_{e}=\left(g^{a}, g^{b}\right)$.

$\operatorname{Enc}\left(p, p k_{e}, m\right)$

1. Parses $p=\left(q, \mathbb{G}, \mathbb{G}_{T}, \mathbf{e}, g\right)$.

2. Selects $r \in \mathbb{Z}_{q}, s \in \mathbb{Z}_{q}$ randomly.

3. Computes $\left(c_{1}, c_{2}, c_{3}\right)=\left(\left(g^{a}\right)^{r},\left(g^{b}\right)^{s}, g^{r+s} m\right)$.

4. Outputs $c=\left(c_{1}, c_{2}, c_{3}\right)$.

$\operatorname{Verify}\left(p, s k_{e}, c\right)$

1. Parses $p=\left(q, \mathbb{G}, \mathbb{G}_{T}, \mathbf{e}, g\right), s k_{e}=(a, b)$, and $c=\left(c_{1}, c_{2}, c_{3}\right)$.

2. Outputs $m=c_{3} /\left(c_{1}^{1 / a} / c_{2}^{1 / b}\right)$.

Theorem 1. [16] Under DL assumption, the linear encryption scheme satisfies the indistinguishability.

\subsection{The Obfuscator for the EBS Functionality}

EBS functionality consists of the blind signature scheme and encryption scheme above. We construct a circuit $C_{p, s k, p k_{e}}$ which contains a common parameter $p$, the signing secret key $s k$ and the public encryption key $p k_{e}$. Note that the important point of obfuscation is how to rerandomize the Enc to make the two results scalar homomorphic. Here, we use the ReRand algorithm, given a cipertext $\left(c_{1}, c_{2}, c_{3}\right)$ and public key $p k_{e}=\left(g^{a}, g^{b}\right)$, to rerandomize the ciphertext $\left(c_{1}, c_{2}, c_{3}\right)$ as following: $\left(c_{1}\left(g^{a}\right)^{r^{\prime}}, c_{2}\left(g^{b}\right)^{s^{\prime}}, c_{3} g^{r^{\prime}+s^{\prime}}\right) \leftarrow$ $\operatorname{ReRand}\left(p, p k_{e},\left(c_{1}, c_{2}, c_{3}\right)\right)$, where $r^{\prime}, s^{\prime} \in \mathbb{Z}_{q}$ are random parameters.

Given a circuit $C_{p, s k, p k_{e}}$, the detail of our obfuscator for the EBS Functionality $O b f_{E B S}$ is as below:

1. Extracts $\left(p, s k, p k, p k_{e}\right)$, where $s k=g_{1}^{x}, p k=g^{g_{1}^{x}}$ and $p k_{e}=\left(g^{a}, g^{b}\right)$.

2. Parses $p=\left(q, \mathbb{G}, \mathbb{G}_{T}, \mathbf{e}, g\right)$.

3. Signer runs $\operatorname{Enc}\left(p, p k_{e}, s k\right) \rightarrow\left(c_{1}, c_{2}, s k^{\prime}\right)=\left(\left(g^{a}\right)^{r},\left(g^{b}\right)^{s}, g^{r+s} g_{1}^{x}\right)$ to obtain a new signing secret keysk $k^{\prime}=g^{r+s} g_{1}^{x}$, computes the corresponding public signing key $p k^{\prime}=\left(g_{1}, g^{g^{r+s} g_{1}^{x}}\right)$, where $y^{\prime}=g^{g^{r+s} g_{1}^{x}}$, and sends $\left(c_{1}, c_{2}\right)$ to User.

4. Signer selects a random parameter $k \in \mathbb{Z}_{q}$, then sends $t=g^{k}$ to User.

5. Randomly chooses $\alpha, \beta \in \mathbb{Z}_{q}$, User counts $\omega^{\prime}=\operatorname{tg}^{\alpha}\left(y^{\prime}\right)^{\beta}, c^{\prime}=H\left(m \| \omega^{\prime}\right)$, and $c^{\prime \prime}=c^{\prime}-\beta$, then transmits $c^{\prime \prime}$ to Signer.

6. Signer gives User $u^{\prime}$, where $u^{\prime}=k-c^{\prime \prime} \cdot s k^{\prime}$. 
7. User gets $\left(c^{\prime}, v^{\prime}\right)=\left(H\left(m|| \omega^{\prime}\right), u^{\prime}+\alpha\right)$.

8. User computes $c_{3}=c_{1}^{1 / a} c_{2}^{1 / b} c^{\prime}$, rerandomizes the ciphertext $\left(c_{1}, c_{2}, c_{3}\right)$ as $C_{1}=$ $\left(c_{1}^{\prime}, c_{2}^{\prime}, c_{3}^{\prime}\right) \leftarrow \operatorname{ReRand}\left(p, p k_{e},\left(c_{1}, c_{2}, c_{3}\right)\right)$

(Note: $\left(c_{1}^{\prime}, c_{2}^{\prime}, c_{3}^{\prime}\right)=\left(\left(g^{a}\right)^{r+r^{\prime}},\left(g^{b}\right)^{s+s^{\prime}}, c^{\prime} g^{r+r^{\prime}+s+s^{\prime}}\right)$.

9. User computes $C_{2} \leftarrow \operatorname{Enc}\left(p, p k, v^{\prime}\right)$. (We define $C_{2}=\left(c_{1}^{\prime \prime}, c_{2}^{\prime \prime}, c_{3}^{\prime \prime}\right)$ ).

10. User outputs the encrypted blind signature $\sigma=\left(C_{1}, C_{2}\right)$.

The output signature $\sigma=\left(C_{1}, C_{2}\right)$ is blind to the Signer, as Signer couldn't recognize either $\left(c^{\prime}, v^{\prime}\right)$ or $(\alpha, \beta)$. But User can verify the signature $\sigma$ by following verification algorithm $V(p, p k, m, \sigma)$ :

1. Computes $c^{\prime}=c_{3}^{\prime} /\left(\left(c_{1}^{\prime}\right)^{1 / a}\left(c_{2}^{\prime}\right)^{1 / b}\right), v^{\prime}=c_{3}^{\prime \prime} /\left(\left(c_{1}^{\prime \prime}\right)^{1 / a}\left(c_{2}^{\prime \prime}\right)^{1 / b}\right), g^{v^{\prime}} y^{\prime c^{\prime}}=\widehat{\omega}$, and $H(m \| \widehat{\omega})=\widehat{c}$.

2. If $\widehat{c}=c^{\prime}$, accepts $\sigma=\left(C_{1}, C_{2}\right)$; otherwise outputs $\perp$.

Obviously, the obfuscation can be executed in polynomial time and has the same functionality compared with the original blind signature. So we omit the two proofs about functionality and polynomial slowdown.

\section{The New Security Definition of the Blind Signature in the Context of EBS}

We modify the above definitions to adapt to our proposals in the context of EBS. As we need to prove the security of blind signature in the presence of the obfuscator we proposed. In this section, we allow the Signer to access the obfuscation circuit as follows:

Definition 5. (Blindness w.r.t. EBS Obfuscator) An encrypted signature scheme EBS = ( $S K G, E K G,(S, U), V)$ w.r.t obfuscator is called blind if for any efficient algorithm $A_{3}$, all sufficiently large $n \in \mathbb{N}$, and every $z \in\{0,1\}^{\text {poly }(n)}$, there exists

$2 \cdot \operatorname{Pr}\left[\begin{array}{l}p \leftarrow \operatorname{Setup}\left(1^{n}\right) ;(p k, s k) \leftarrow S K G(p) ;\left(p k_{e}, s k_{e}\right) \leftarrow E K G(p) ; \\ C^{\prime} \leftarrow \operatorname{Obf}\left(C_{p, s k, p k_{e}}\right) ; \\ b \leftarrow\{0,1\} ;\left(\sigma_{0}, \sigma_{1}\right) \leftarrow A_{3}^{<\cdot, U\left(p k, m_{b}\right)>^{1},<\cdot, U\left(p k, m_{1}-b\right)>^{1}}\left(p, p k, p k_{e}, C^{\prime}, z\right) ; \\ b^{*} \leftarrow A_{3}\left(\sigma_{0}, \sigma_{1}\right) ; \\ b=b^{*} .\end{array}\right]-1 \leqslant \frac{1}{p(n)}$

where $A_{3}$ is the malicious Signer and $U$ is the honest user. If $\sigma_{0}=\perp$ or $\sigma_{1}=\perp$, then the Signer is not informed about the other signature.

Definition 6. (One-more Unforgeability w.r.t. EBS Obfuscator) An EBS scheme ( $S K G, E K G$, $(S, U), V)$ is unforgetable if for any efficient algorithm $A_{4}$ (the malicious user), every polynomial $p(\cdot)$, all sufficiently large $n \in \mathbb{N}$, and every $z \in\{0,1\}^{\text {poly }(n)}$, there exists

$$
\operatorname{Pr}\left[\begin{array}{l}
p \leftarrow \operatorname{Setup}\left(1^{n}\right) ;(p k, s k) \leftarrow S K G(p) ;\left(p k_{e}, s k_{e}\right) \leftarrow E K G(p) ; \\
C^{\prime} \leftarrow \operatorname{Obf}\left(C_{p, s k, p k_{e}}\right) ; \\
\left(\left(m_{1}^{*}, \sigma_{1}^{*}\right), \ldots,\left(m_{k+1}^{*}, \sigma_{k+1}^{*}\right)\right) \leftarrow A_{4}^{\left.\ll S_{p, s k}\right\rangle^{k}}\left(p, p k, p k_{e}, C^{\prime}, z\right) ; \\
\text { if } m_{i}^{*} \neq m_{j}^{*} \text { for } i \neq j ; \\
V\left(p, p k, m_{i}^{*}, \sigma_{i}^{*}\right)=\text { Accept for all } i ; \text { then return } 1 .
\end{array}\right] \leqslant \frac{1}{p(n)}
$$


where $S_{p, s k}$ is the signing oracle (circuit).

Definition 7. (ACVBP w.r.t Dependent Oracles) Let $T(C)$ be a set of oracles dependen$t$ on the circuit $C$. A circuit obfuscator Obf for $C$ satisfies the ACVBP w.r.t dependent oracle set $T$ if the following condition holds: There exists a PPT oracle machine $S$ ( simulator) such that, for every PPT oracle machine $D$ (distinguisher), every polynomial $p(\cdot)$, all sufficiently large $n \in \mathbb{N}$, and every $z \in\{0,1\}^{\text {poly }(n)}$,

$\left|\operatorname{Pr}\left[\begin{array}{l}C \leftarrow C_{n} ; \\ C^{\prime} \leftarrow \operatorname{Obf}(C) ; \quad: b=1 \\ b \leftarrow D^{\ll C, T(C) \gg}\left(C^{\prime}, z\right) .\end{array}\right]-\operatorname{Pr}\left[\begin{array}{l}C \leftarrow C_{n} ; \\ C^{\prime \prime} \leftarrow S^{\ll C \gg}\left(1^{n}, z\right) ; \\ b \leftarrow D^{\ll C, T(C) \gg\left(C^{\prime \prime}, z\right) .} \quad: b=1\end{array}\right]\right| \leqslant \frac{1}{p(n)}$

where $D^{\ll C, T(C) \gg}$ means that $D$ has sampling access to all oracles contained in $T(C)$ in addition to $C$.

\section{The Security of Special EBS Obfuscator}

In this section, we attribute the the security of special EBS obfuscator to DL assumption and the random oracle model. Although our obfuscation can remove the random oracle in theory, there still have no effective methods to do so. The reason why we prove it in random oracle model is that the signature scheme we choose is secure in random model, which is a inherent property of the original signature scheme.

At first, we will prove the completeness property of our special EBS obfuscator. Informally, the signature is complete if for any message $m$, verification algorithm $V(p, p k, m, \sigma)$ always set up, i.e., the probability: $P r_{V(p, p k, m, \sigma)}=1$.

Lemma 1. The EBS obfuscation is complete.

Proof. Once the user receives the signature $\sigma=\left(C_{1}, C_{2}\right)$, he finishes the following proceeds in a polynomial reduction:

1. Computes $c^{\prime}=c_{3}^{\prime} /\left(\left(c_{1}^{\prime}\right)^{1 / a}\left(c_{2}^{\prime}\right)^{1 / b}\right)$.

2. Computes $v^{\prime}=c_{3}^{\prime \prime} /\left(\left(c_{1}^{\prime \prime}\right)^{1 / a}\left(c_{2}^{\prime \prime}\right)^{1 / b}\right)$.

According to the verification algorithm, he has $g^{v^{\prime}}\left(y^{\prime}\right)^{c^{\prime}}=g^{u^{\prime}+\alpha} g^{g_{1}^{x} c^{\prime}}=g^{u^{\prime}+\alpha+g_{1}^{x} c^{\prime}}$. As $u^{\prime}=k-c^{\prime \prime} \cdot s k^{\prime}$ and $c^{\prime \prime}=c^{\prime}-\beta$, he obtains the equation $u^{\prime}+\alpha+g_{1}^{x} c^{\prime}=k+\alpha+\beta s k^{\prime}$. Thus, $g^{v^{\prime}} y^{\prime c^{\prime}}=g^{k} g^{\alpha} g^{\beta s k^{\prime}}$. Since $t=g^{k}$ and $y^{\prime}=g^{s k^{\prime}}$, he gets $g^{v^{\prime}} y^{\prime c^{\prime}}=t g^{\alpha} g^{\beta}=\omega^{\prime}$. Then, the equation $H\left(m \| \omega^{\prime}\right)=c^{\prime}$ must be established. We outcome the completeness of EBS obfuscation.

Theorem 2. Under DL assumption, for the EBS obfuscator and two messages $m_{0}, m_{1}$ selected by the malicious Signer $A_{3}$, the distributions of $\sigma_{0}$ and $\sigma_{1}$ are computationally indistinguishable.

Proof. The blindness of EBS obfuscator follows directly from the hardness of DL assumption in the group $\mathbb{G}$. More formally, we show that if an adversary $A_{3}$ can distinguish the signatures $\left(\sigma_{0}, \sigma_{1}\right)$ of two message $m_{0}$ and $m_{1}$ under $s k$ with non-negligible 
probability, then we construct an adversary $A^{\prime}$ that will break the DL assumption with advantage $\epsilon$ as well.

At first, we analyze the result of EBS obfuscator: we get $\sigma=\left(C_{1}, C_{2}\right)=\left(\left(g^{a}\right)^{r+r^{\prime}}\right.$, $\left.\left(g^{b}\right)^{s+s^{\prime}}, c^{\prime} g^{r+r^{\prime}+s+s^{\prime}},\left(g^{a}\right)^{r^{\prime \prime}},\left(g^{b}\right)^{s^{\prime \prime}}, v^{\prime} g^{r^{\prime \prime}+s^{\prime \prime}}\right)$, where $r, s, r^{\prime}, s^{\prime}, r^{\prime \prime}, s^{\prime \prime}$ are all random parameters. Through the process of obfuscation above, we have $c^{\prime}=H\left(m \| \omega^{\prime}\right), v^{\prime}=$ $k-c^{\prime} \cdot s k^{\prime}+\beta \cdot s k^{\prime}+\alpha$, where $k, \alpha, \beta$ are random and $\omega^{\prime}=g^{k} g^{\alpha}\left(y^{\prime}\right)^{\beta}$. So when the secret key $s k^{\prime}$ is fixed, $v^{\prime}$ depends on the value of $c^{\prime}$ (i.e, $v^{\prime}$ and $c^{\prime}$ are linearly dependent). Thus the value of $C_{2}$ relies on $c^{\prime}$. Since $C_{1}$ and $C_{2}$ have the same form, we can only consider $C_{1}$ in the following work $\left(C_{2}\right.$ also has the same result, we omit it here $)$. Let $\widehat{s}=s+s^{\prime}$, $\widehat{r}=r+r^{\prime}$, so we have $C_{1}=\left(g^{\widehat{r}}, g^{\widehat{s}}, g^{\widehat{r}+\widehat{s}}\right)$.

$A^{\prime}$ works as follows:

- $A^{\prime}$ receives as input a tuple $\left(g,(a, b), B=g^{\widehat{r}}, K=g^{\widehat{s}}, W\right)$ where $g$ is a random generator of the group $\mathbb{G}$ and $r, s$ are random exponents. The goal of $A^{\prime}$ is to determine whether $W=g^{\hat{r}+\hat{s}}$.

- $A^{\prime}$ picks a random generator $g$ of group $\mathbb{G}$.

- On receiving two messages $m_{0}$ and $m_{1}$ from $A_{3}, A^{\prime}$ flips a bit $b$ randomly and sends the signature $\sigma_{b}:=\left(\left(g^{a}\right)^{\hat{r}},\left(g^{b}\right)^{\hat{s}}, c_{b} W\right)$ as the signature of $m_{b}$ to $A_{3}$.

- $A_{3}$ replies with a bit $b^{*} . A^{\prime}$ simply outputs 1 if $b=b^{*}$ (i.e., guessing that $W=$ $g^{\widehat{r}+\widehat{s}}$ ); otherwise outputs a random bit(i.e., $W$ is a random parameter).

It is easy to see that when $W$ is random, the signature $\sigma_{b}$ is independent of $b$ and hence the success probability of $A_{3}$ is exactly $\frac{1}{2}$ in this case. When $W=g^{\hat{r}+\hat{s}}$, the signature $\sigma_{b}$ has the same distribution as the result of EBS obfuscator. According to the assumption, the adversary $A_{3}$ has advantage at least $\epsilon$. That is, $A^{\prime}$ succeeds in determining whether $W=g^{\hat{r}+\hat{s}}$ with non-negligible advantage, $A^{\prime}$ breaks the DL assumption.

Theorem 3. [15] The blind signature is one-more unforgeable if discrete logarithm is hard.

Theorem 4. Let $T\left(C_{p, s k, p k_{e}}\right)$ be $S_{p, s k}$. If the EBS obfuscator satisfies ACVBP w.r.t dependent oracle set $T$, then the one-more unforgeability $(O M U)$ w.r.t the EBS functionality implies the one-more unforgeability w.r.t EBS obfuscator.

Proof. We show that, if there exists an adversary $A_{4}$ to break the OMU w.r.t Obf when the OMU w.r.t EBS is satisfied, then it will contradict the ACVBP w.r.t dependent oracle set $T$ of EBS obfuscator. Let the distinguisher $D$ has sample access to $T\left(C_{p . s k, p k_{e}}\right)$ to check whether $A_{4}$ succeeds in breaking OMU w.r.t Obf.

1. Inputs a circuit $C$ (either an obfuscated circuit or a simulated circuit) and an auxiliaryinput $z$.

2. Extracts $\left(p, p k, p k_{e}\right)$ through sampling access to $C_{p, p k, p k_{e}}$.

3. Samples access to $S_{p, s k}$ at most $k$ times $\left(\left(m_{1}^{*}, \sigma_{1}^{*}\right), \ldots,\left(m_{k}^{*}, \sigma_{k}^{*}\right)\right) \leftarrow A_{4}^{\left.\ll S_{p, s k}\right\rangle^{k}}(p, p k$, $\left.p k_{e}, C, z\right)$ to simulate $\left(m_{k+1}^{*}, \sigma_{k+1}^{*}\right)$.

4. $V\left(p, p k, m_{k+1}^{*}, \sigma_{k+1}^{*}\right)=$ Accept for $m_{k+1} \neq m_{i}$ where $i \in\{1, k\}$.

If $C$ is an obfuscated circuit, then the probability $D$ outputs 1 which is equal to the probability that $A_{4}$ breaks OMU w.r.t Obf, which is non-negligible by the assumption. And 
if $C$ is a simulated circuit, then the probability that $D$ outputs 1 is negligible, otherwise, $A_{4}$ can break the OMU w.r.t EBS functionality. So the probability which ACVBP established is non-negligible. Hence it will contradict the ACVBP w.r.t dependent oracle set $T$ of EBS obfuscator. Theorem is established.

Theorem 5. Let $T\left(C_{p, s k, p k_{e}}\right)$ be $S_{p, s k}$. The EBS obfuscator satisfies ACVBP w.r.t dependent oracle set $T$ under DL assumption.

Proof. According to the EBS obfuscator we proposed, the security proof of obfuscator containing an interactive process between Signer and User is a little different from the previous work. We use the variant of Hada's proof method. At first, we construct a simulator $S$ to simulate the behaves of the obfuscated circuit; the execution process is as follows(Note that the value $\left(p, p k, p k_{e}\right)$ is easy to get through sampling access to $C_{p, p k, p k_{e}}$. So we mainly focus on $\left(s k^{\prime},\left(c_{1}, c_{2}\right)\right)$.):

1. Inputs the security parameter $1^{n}$ and an auxiliary-input $z$.

2. Extracts $\left(p, p k, p k_{e}\right)$ through sampling access to $C_{p, p k, p k_{e}}$.

3. Parses $p=\left(q, \mathbb{G}, \mathbb{G}_{T}, \mathbf{e}, g\right)$ and $p k=\left(g_{1}, g^{g_{1}^{x}}\right)$.

4. Randomly selects Junk $\leftarrow \mathbb{G}$.

5. Computes $c_{1}, c_{2}, c_{3} \leftarrow \operatorname{Enc}\left(p, p k_{e}, J u n k\right)$ and sets $s k^{\prime}=c_{3}$.

6. Outputs $\left(s k^{\prime},\left(c_{1}, c_{2}\right)\right)$.

And then we consider the worst case that the interactive values are captured by adversary already, i.e., the value of $k, t, c^{\prime \prime}, u^{\prime}, v^{\prime}, \omega^{\prime}$ are $\operatorname{known}\left(\omega^{\prime}\right.$ can get by computing $g^{v^{\prime}} y^{c^{\prime}}$ ), we proved the output distribution of $S$ is indistinguishable from the real distribution $\left(C_{1}, C_{2}\right)$ for any PPT distinguisher. In particular, when the distinguisher is permitted to sampling access to $C S=\left\{C_{p, s k, p k_{e}}, S_{p, s k}\right\}$, assume the probability that a distinguisher $D^{\ll C, S 》}$ distinguishes the two output distributions above is nonnegligible. In other word, the probability of the following formula is non-negligible. And let $z=\left(k, t, c^{\prime \prime}, u^{\prime}, v^{\prime}, \omega^{\prime}\right)$ be the auxiliary-input, we have:

Real one:

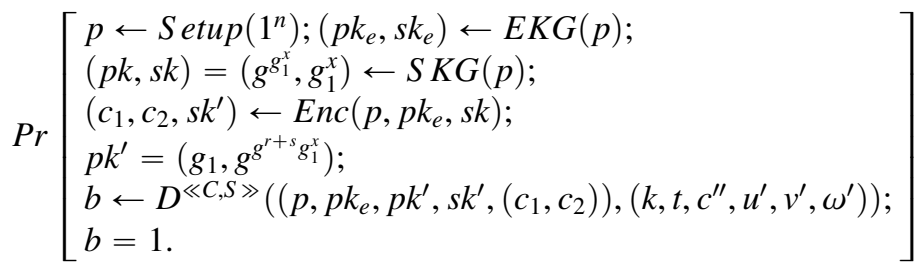

Junk one:

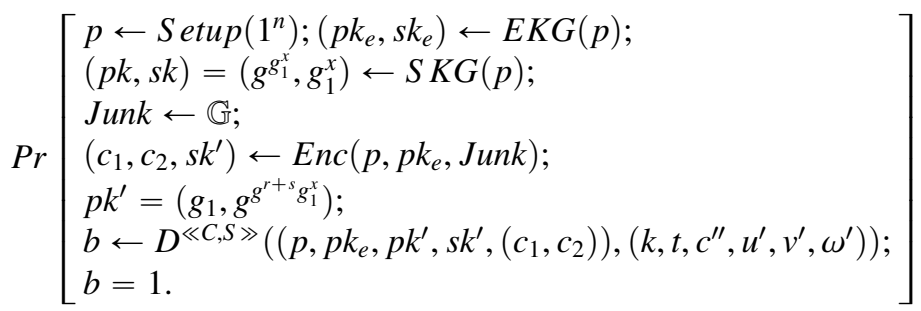


Third we construct an adversary $\left(A_{1}, A_{2}\right)$ to break the indistinguishability of the linear encryption scheme. $A_{1}$ produces a message pair $\left(m_{1}, m_{2}\right)=($ sk, Junk) and an associated hint $h=p k$. Given a ciphertext $c$ (of either $m_{1}$ or $m_{2}$ ), $A_{2}$ distinguishes the results of $m_{1}$ and $m_{2}$ by distinguisher $D$ as follows:

1. Parses $p=\left(q, \mathbb{G}, \mathbb{G}_{T}, \mathbf{e}, g\right)$ and $p k_{e}$, cipertext $c$ and auxiliary $z=\left(k, t, c^{\prime \prime}, u^{\prime}, v^{\prime}, \omega^{\prime}\right)$.

2. Get the output $m_{1}, m_{2}$ of $A_{1}, h=p k=g^{g_{1}^{x}}$ and $c=\left(c_{1}, c_{2}, s k^{\prime}\right)$, let $p k^{\prime}=g^{c_{3}}$.

3. Simulates $D^{\ll C, S 》}\left(\left(p, p k_{e}, p k^{\prime}, s k^{\prime},\left(c_{1}, c_{2}\right)\right),\left(k, t, c^{\prime \prime}, u^{\prime}, v^{\prime}, \omega^{\prime}\right)\right)$.

4. Outputs the result of $D$.

If $c$ is a ciphertext of $m_{1}$, then the probability $A_{2}$ outputs 1 which is equal to the first probability, otherwise, it is equal to the later probability. According to the Theorem 1 , the difference of the two probability above is negligible which contradicts to the assumption. Theorem is established.

\section{Conclusion}

A new functionality for obfuscation has been proposed in this paper under DL assumption and the hardness of discrete logarithm. Following Hohenberger and Hada's steps, we present two new security definitions and our scheme is a further application, which not only protects the Signer's secret key from revealing, but also keeps the signature blinding from the Signer. This functionality is very useful in E-Cash and E-Vote. At the same time, our scheme resists different PPT adversaries and satisfies ACVBP w.r.t dependent oracle property. Furthermore, we will continue to fucusing the research and application of obfuscation.

\section{References}

1. Boaz Barak,Oded Goldreich, Russell Impagliazzo, Steven Rudich, Amit Sahai, Salil Vadhan and Yang K. On the (im)possibility of obfuscating program.In CRYPTO, Advances in Cryptology-CRYPTO 2001, 21st Annual International Cryptology Conference, Santa Barbara, California,USA, August 19-23, 2001. Proceedings,pages 1-18, 2001.

2. Shafi Goldwasser and Yael Tauman Kalai. On the impossibility of obfuscation with auxiliary input. In FOCS, 46th Annual IEEE Symposium on Foundations of Computer Science (FOCS) 2005, 23-25 October 2005, Pittsburgh, PA, USA. Proceedings, pages 553-562, 2005.

3. Hoeteck Wee. On obfuscating point functions. In STOC, Proceedings of the 37th Annual ACM Symposium on Theory of Computing, Baltimore, MD, USA, May 22-24, 2005, pages 523-532, 2005.

4. Dennis Hofheinz, John Malone-Lee, and Martijn Stam. Obfuscation for cryptographic purposes. In TCC 2007, Theory of Cryptography, 4th Theory of Cryptography Conference, TCC 2007, Amsterdam, The Netherlands, February 21-24, 2007. Proceedings, pages 214-232, 2007.

5. Nir Bitansky and Ran Canetti. On strong simulation and composable point obfuscation. In CRYPTO, Advances in Cryptology Conference, Santa Barbara, California, USA, August 19-23, 2001. Proceedings, pages 1-18, 2001. 
6. Ran Canetti. Towards realizing random oracles: Hashing functions that hide all partial information. In CRYPTO, Advances in Cryptology-CRYPTO'97, 17th Annual International Cryptology Conference, Santa Barbara, California, USA, August 17-21, 1997. Proceedings, pages 455-469, 1997.

7. Ran Canetti, Daniele Miccianico, and Omer Reingold. Perfectly one-way probabilistic hash functions. In STOC, pages 72-89, 2010.

8. Ben Lynn, Manoj Prabhakaran, and Amit Sahai. Positive results and techniques for obfuscation. In EUROCRYPT, Advances in Crytology-EUROCRYPT 2004, International Conference on the Theory and Applications of Cryptographic Techniques, Interlaken, Switzerland, May 2-6, 2004. Proceedings, pages 20-39, 2004.

9. Ran Canetti and Ronny Ramzi Dakdouk. Obfuscating point functions with multibit output. In EUROCRYPT, Advances in Cryptology-EUROCRYPT 2008, 27th Annual International Conference on the Theory and Applications of Cryptographic Techniques, Istanbul, Turkey, April 13-17, 2008. Proceedings, pages 92-112, 2010.

10. Susan Hohenberger, Guy N.Rothblum, Abhi Shelat, and Vinod Vaikuntanathan. Securely obfuscating re-encryption. In TCC, Theory of Cryptography, 4th Theory of Cryptography Conference, TCC 2007, Amsterdam, The Netherlands, February 21-24, 2007. Proceedings, pages 233-252, 2007.

11. Satoshi Hada. Secure obfuscation for encrypted signatures. In EUROCRYPT, Advances in Cryptology-EUROCRYPT 2010, 29th Annual International Conference on the Theory and Applications of Cryptographic Techniques, French Riviera, May 30-June 3, 2010. Preceedings, pages 92-112, 2010.

12. Nishanth Chandran, Melissa Chase and Vinod Vaikuntanathan. Functional Re-encryption and Collusion-Resistant Obfuscation. In TCC, 9th Theory of Cryptography Conference, TCC 2012, Taormina, Sicily, Italy, March 19-21, 2012. Proceedings, pages 404-421, 2012.

13. David Chaum. Blind Signatures for Untraceable Payments. In R.L.Rivest, A.Sherman, and D.Chaum, editors.In CRYPTO 82, New York. Preceedings pages 199-203, 2010.

14. Tatsuaki Okamoto. Provably Secure and Practical Identification Schemes and Corresponding Signature Schemes. In CRYPTO, Advances in Cryptology ł CRYPTO92, 12th Annual International Cryptology Conference Santa Barbara, California, USA August 16C20. Proceedings pages 31-53, 1992.

15. Claus Peter Schnorr. Enhancing the Security of Perfect Blind DL-Signatures. In Information Sciences, Volume 176, Issue 10, 22 May 2006. Preceedings Pages 1305C1320, 2006.

16. Dan Boneh, Xavier Boyen, and Hovav Shacham. Short Group Signatures. In CRYPTO, Advances in Cryptology C CRYPTO 2004, 24th Annual International Cryptology Conference, Santa Barbara, California, USA, August 15-19, 2004. Proceedings pages 41-55. 